\title{
Medicinal Gas Dosage Form Category
}

National Cancer Institute

\section{Source}

National Cancer Institute. Medicinal Gas Dosage Form Category. NCI Thesaurus. Code C148452.

A type of gaseous pharmaceutical dose form consisting of a pure substance or mixture of substances that exist in the form of a gas at normal atmospheric pressure and room temperature. 